\title{
PURIFICAÇÃO DE ÓLEO DE FRITURA
}

\author{
PEDRO RAMOS COSTA NETO * \\ RENATO JOÃO SOSSELA DE FREITAS **
}

\begin{abstract}
Neste trabalho verificou-se a eficiência de diferentes adsorventes na purificação de óleo de soja usado em frituras. Foi verificado, igualmente, a eficiência de mistura de carvão ativo, tonsil e bauxita na purificação de óleo que havia

sido utilizado por $18,40,68$ e 79 h. A reativação e reutilização da mistura adsorvente também foi testada e mostrou-se viável.
\end{abstract}

\section{INTRODUÇÃO}

Os óleos e gorduras depois de usados em frituras em lanchonetes e restaurantes comerciais tornam-se resíduos com pouca possibilidade de reutilização. Em alguns casos, são usados na produção de sabões, mas, de modo geral, vão parar no esgoto, contribuindo para agravar os problemas do meio ambiente.

A purificação de óleo de fritura tem sido tentada por diversos pesquisadores $(2,4,5,7,8,9,10,11,12,13,15,16,18$, $19,20,21,22)$. Entre as substâncias utilizadas nas purificações destacam-se os adsorventes carvão ativo, terras clarificantes e sílica gel. Essas substâncias removem produtos de decomposição não voláteis e são inócuas ao óleo quando usadas diretamente. Removem os tocoferóis (11), entretanto, segundo BOSKOU (3), os antioxidantes naturais são degradados durante o processo de fritura devido à alta temperatura a qual são submetidos.

* Professor da Área de Alimentos do Centro Federal de Educação Tecnológica do Paraná/Uned-Medianeira.

* * Professor do Departamento de Tecnologia Química da Universidade Federal do Paraná.

Considerando que no Brasil não existe legislação que estabeleça o tempo de reutilização de óleo em fritura por imersão, em algumas lanchonetes e restaurantes comerciais a troca só é feita a cada três meses (6). Purificar óleo assim 
oxidado não é viável, mas, a partir do momento que houver condições de remover algumas impurezas desse óleo, através de um processo simples, em determinados intervalos, com certeza a qualidade do produto frito seria melhorada.

Neste sentido, o presente trabalho apresenta os seguintes objetivos:

1) Verificar a eficiência de materiais adsorventes na clarificação e redução da acidez de óleo de soja usado em frituras mútiplas (massas e carnes), por longos períodos, visando deixar estas características equivalentes às de um óleo novo.

2) Verificar a viabilidade de purificar óleo aquecido em diferentes intervalos, utilizando mistura de adsorventes (carvão ativo, tonsil e bauxita).

3) Verificar a possibilidade de reativar e reutilizar a mistura de adsorventes usada.

\section{MATERIAL E MÉTODOS}

\section{* óleo de soja}

o óleo foi adquirido no comércio local (Curitiba-PR), em bombona de 50 L e usado em fritadeira elétrica de lanchonete comercial na fritura de alimentos durante 18, 40, 68 e $79 \mathrm{~h}$ consecutivas, com períodos diários de aquecimento ininterrupto de 12 a $14 \mathrm{~h}$. Nesse tempo houve reposição de 65\% de óleo novo sobre o óleo em uso.

\section{* Adsorventes}

Carvão ativo, sílica amorfa, diatomita ativada, terra fuller, filtrol, tonsil, decosil, fulmont, argila atapulgita e bauxito ativado (bauxita).

Os adsorventes foram cedidos gentilmente pela Companhia Paranaense de Energia Elétrica (COPEL) e pela Fábrica Brasileira de Catalisadores (FBC).

\section{*Análises físico-químicas}

A cor foi determinada por espectrofotometria segundo BEADLE et al. (1) e a acidez, em ácido olêico, segundo normas analíticas do IAL (17).

\section{* Purificação}

$\mathrm{Na}$ verificação da eficiência dos adsorventes, os mesmos foram utilizados na forma granulada (12 x 24 mesh), em coluna de vidro $(2 \times 50 \mathrm{~cm})$. Uma parte do óleo usado por

79 h foi eluído na relação de 1,0 g de adsorvente para

$1,5 \mathrm{~mL}$ de óleo, a temperatura ambiente. Enquanto os óleos usados por 18, 40, 68 e 79 h foram tratados com mistura de 
carvão ativo-20\%, tonsil-40\% e bauxita-40\%, em granulometria superior a 95\% na malha 325 mesh, com uma hora de agitação a $80^{\circ} \mathrm{C}$ e filtrado por gravidade.

\section{* Reativação}

A mistura adsorvente (carvão ativo, tonsil e bauxita) usada na purificação foi lavada com hexano até a retirada completa do óleo impregnado, sendo uma parte reativada em estufa a $100^{\circ} \mathrm{C}$ e a outra em mufla a $500^{\circ} \mathrm{C}$ por $3 \mathrm{~h}$.

\section{RESULTADOS E DISCUSSÃO}

\section{* Eficiência dos adsorventes}

$\mathrm{Na}$ Tabela 1 mostram-se os resultados do óleo usado por $79 \mathrm{~h}$ tratado em coluna com diferentes materiais adsorventes. A granulometria dos materiais clarificantes é importante na eluição e retenção do óleo tratado, de modo que quanto maior for o tamanho das partículas, menor será a área de contato e bastante menor o poder clarificante do material (15).

o carvão ativo, a sílica amorfa e a diatomita ativada não foram eficientes na redução da cor e diminuiram muito pouco a acidez, considerando a elevada relação material adsorvente e óleo $(1: 1,5)$. O carvão ativo remove do óleo oxidado hidroperóxidos, ácidos graxos livres, aldeídos, cetonas, cor e odor $(2,6,7,10)$; o mesmo faz a sílica amorfa $\mathrm{Com} \mathrm{MgO}_{2}$ (16) .

Os materiais terra fuller, filtrol, tonsil, decosil e fulmont reduziram parcialmente a cor. O tonsil atuou com maior eficiência, embora a cor obtida não possa ser comparada com a cor do óleo novo. O tonsil é normalmente usado no refino de óleo bruto, destinado a produção de margarinas.

A argila atapulgita e a bauxita reduziram a acidez do óleo usado a patamares equivalentes aos do óleo novo, tendo a primeira reduzido significativamente a cor. A argila atapulgita é constituída por $66 \%$ de $\mathrm{SiO}_{2}$ e $12 \%$ de $\mathrm{Al}_{2} \mathrm{O}_{3}$, enquanto a bauxita possui 78\% de $\mathrm{Al}_{2} \mathrm{O}_{3}$ e $8 \%$ de $\mathrm{SiO}_{2}$. 
TABELA 1 - ÓLEO USADO (79 h), TRATADO EM COLUNAS COM DIFERENTES ADSORVENTES

\begin{tabular}{l|c|c|c}
\hline ADSORVENTES & $\begin{array}{c}\text { CONCENTRAÇÃO } \\
\text { (g/mL) }\end{array}$ & $\begin{array}{c}\text { COR } \\
\text { (espectrofotometria) }\end{array}$ & $\begin{array}{c}\text { ACIDEZ } \\
\text { (\% em olêico) }\end{array}$ \\
\hline Óleo novo & ----- & 15,00 & 0,086 \\
\hline Óleo usado (79h) & ----- & 123,66 & 0,800 \\
\hline Carvão ativo & $1: 1,5$ & 89,17 & 0,470 \\
\hline Sílica amorfa & $1: 1,5$ & 71,82 & 0,440 \\
\hline Diatomita ativada & $1: 1,5$ & 80,00 & 0,290 \\
\hline Terra fuller & $1: 1,5$ & 66,42 & 0,410 \\
\hline Filtrol & $1: 1,5$ & 52,74 & 0,490 \\
\hline Tonsil & $1: 1,5$ & $\mathbf{4 7 , 9 5}$ & 0,370 \\
\hline Decosil & $1: 1,5$ & 59,99 & 0,500 \\
\hline Fulmont & $1: 1,5$ & 64,00 & 0,440 \\
\hline Argila atapulgita & $1: 1,5$ & 49,40 & $\mathbf{0 , 0 6 5}$ \\
\hline Bauxita & $1: 1,5$ & 72,57 & $\mathbf{0 , 0 7 2}$ \\
\hline
\end{tabular}

\section{* Mistura adsorvente}

Na Tabela 2, observa-se que o óleo usado por 18, 40, 68 e 79 h, tratado com a mistura de adsorventes em pó, precisou respectivamente de 17,$5 ; 20 ; 30$ e $40 \mathrm{~g} / 100 \mathrm{~mL}$ para reduzir a acidez a menos de $0,1 \%$, quando ficou equivalente à de óleo de soja novo, conforme a legislação (14). O mesmo não aconteceu em relação a cor. Para melhorar a clarificação seria necessário aumentar o tempo de residência, que nas condições desse experimento provocaria a oxidação do óleo em tratamento (6).

\section{* Reativação}

Observa-se na Figura 1 que o óleo usado por 79 h, tratado com mistura de adsorventes reutilizada, a qual foi reativada em estufa (4) e mufla (5), apresentou valores diferentes de acidez e cor em relação ao óleo tratado com adsorvente que não havia sido reutilizado (3). Nos dois experimentos a cor variou muito pouco e a acidez do óleo tratado com o adsorvente reativado em estufa foi superior à da amostra em branco (2), indicando que havia impurezas retidas e que não foram volatilizadas a $100^{\circ} \mathrm{C}$. A ativação na mufla a $500^{\circ} \mathrm{C}$ foi melhor, porém, ainda insuficiente para baixar a acidez do óleo, ao nível conseguido anteriormente com a mistura nova, 
quando usada na mesma proporção (40 g/100 mL). É provável que se fosse prolongado o aquecimento ou aumentada a temperatura, ter-se-ia ativação mais eficiente.

TABELA 2 - ÓLEO USADO POR 18, 40,68 E 79 h, TRATADO COM MISTURA DE ADSORVENTES PULVERIZADOS (CARVÃO ATIVO, TONSIL E BAUXITA)

\begin{tabular}{|c|c|c|c|}
\hline AMOSTRAS & $\begin{array}{c}\text { CONCENTRAÇÃO } \\
(\mathrm{g} / 100 \mathrm{~mL}) \\
\end{array}$ & $\begin{array}{c}\text { COR } \\
\text { (espectrofotometria) } \\
\end{array}$ & $\begin{array}{c}\text { ACIDEZ } \\
\text { (\%, em olêico) } \\
\end{array}$ \\
\hline Branco (novo) & ------- & 15,90 & 0,086 \\
\hline Branco (18h) & ------- & 24,27 & 0,430 \\
\hline Mistura & 5,0 & 20,41 & 0,270 \\
\hline Mistura & 10,0 & 17,91 & 0,180 \\
\hline Mistura & 15,0 & 14,75 & 0,120 \\
\hline Mistura & 17,5 & 14,16 & 0,090 \\
\hline Branco $(40 \mathrm{~h})$ & ------- & 33,80 & 0,430 \\
\hline Mistura & 10,0 & 23,10 & 0,180 \\
\hline Mistura & 20,0 & 21,58 & 0,090 \\
\hline Branco (68 h) & ------- & 55,44 & 0,550 \\
\hline Mistura & 10,0 & 50,87 & 0,320 \\
\hline Mistura & 20,0 & 50,65 & 0,210 \\
\hline Mistura & 30,0 & 41,86 & 0,090 \\
\hline Branco (79 h) & ------- & 123,66 & 0,800 \\
\hline Mistura & 10,0 & 74,57 & 0,440 \\
\hline Mistura & 20,0 & 65,24 & 0,250 \\
\hline Mistura & 30,0 & 59,58 & 0,140 \\
\hline Mistura & 40,0 & 55,62 & 0,080 \\
\hline
\end{tabular}




\section{FIGURA 1 - ÓLEO USADO (79 h) TRATADO COM MISTURA ADSORVENTE REATIVADA}

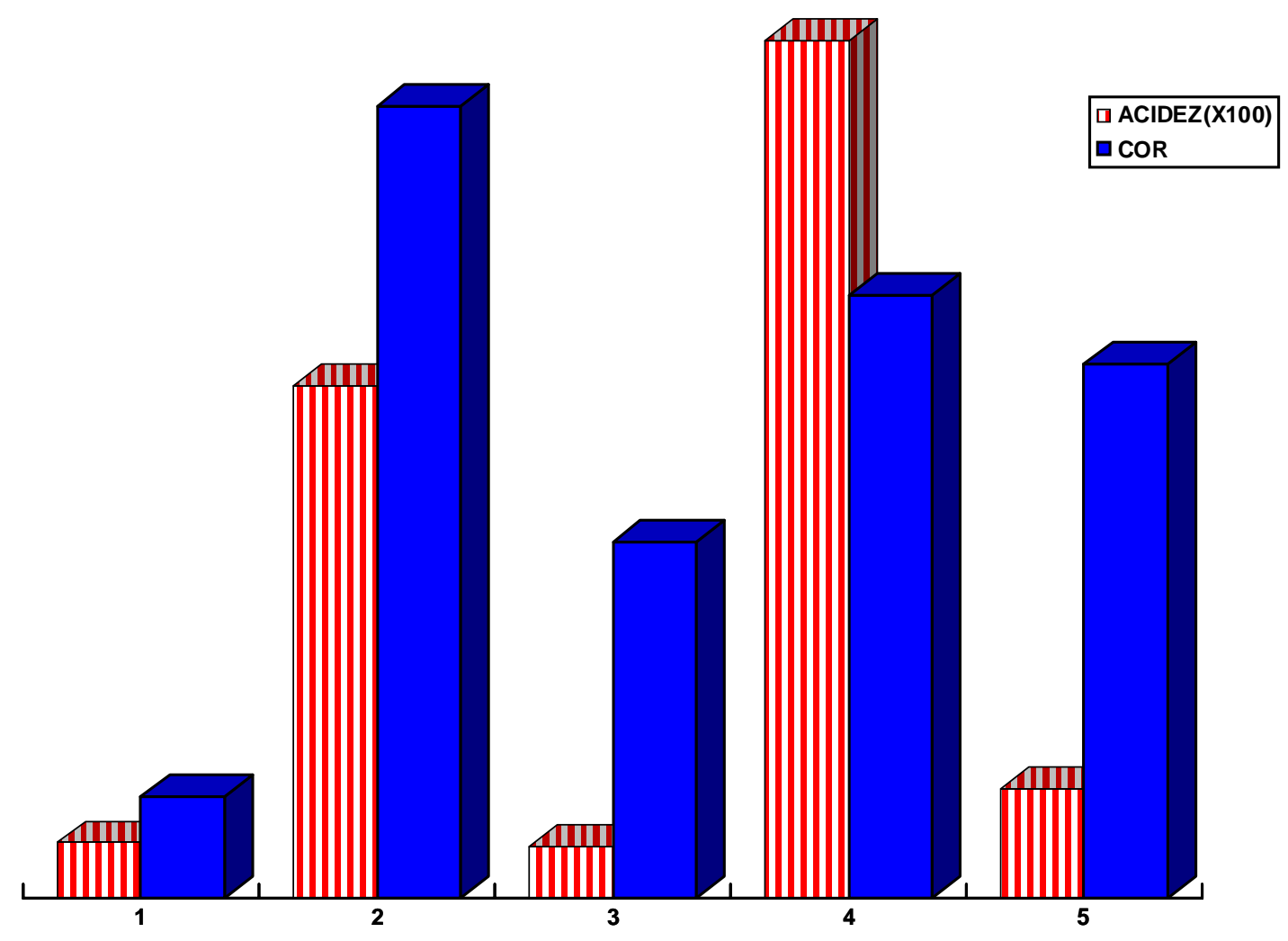

1 - óleo novo.

2 - Óleo usado (79 h)

3 - Óleo usado tratado com mistura adsorvente nova.

4 - Óleo usado tratado com mistura adsorvente reativada a $100^{\circ} \mathrm{C}$.

5 - Óleo usado tratado com mistura adsorvente reativada a $500^{\circ} \mathrm{C}$.

\section{CONCLUSÃO}

* A purificação do óleo usado com diferentes adsorventes granulados mostrou que o tonsil removeu principalmente a cor, a argila atapulgita diminuiu a cor e a acidez, e a bauxita reduziu a acidez. Os demais materiais não atuaram com eficiência.

* A purificação do óleo com diferentes tempos de uso, com mistura constituída por carvão ativo, tonsil e bauxita, reduziu a acidez para níveis equivalentes aos de óleo novo. Entretanto, a quantidade de adsorvente para esta redução foi elevada. 
* A mistura de adsorvente usada, depois de reativada, mostrou-se eficiente na reutilização para purificação do óleo, principalmente na redução da acidez.

\section{Abstract}

It was tested the efficiency of different adsorvents in the purification of soybean oil used in fryings. It was also confirmed the efficiency of a mixture of activated carbon, tonsil and bauxite in the purification of the oil used for 18, 4068 and 79 hours, respectively. The reactivation and reutilization of the adsorvent mixture were also tested and good results were obtained.

\section{REFERÊNCIAS BILBLIOGRÁFICAS}

1 BEADLE, J.B., JUST, D.E., MORGAN, R.E., REINERS, R.A. Compositon of corn oil. JAOCS, v. 12, p. 90-95, 1935.

2 BOKI, K., WADA, T., OHNO, S. Effects of filtration trough activated carbons on peroxides, thiobarbituric acid and carbonyl values of autoxidized soybean oil. JAocs, Osaka, v. 68, n. 8, p. 561-565, 1991.

3 BOSKOU, D. Stability of frying oils. In: FRYING of food. Chichester : Horwood, 1988. p.174-184.

4 BURTSEV, V.A., LOSITSKII, V.V., TARAN, A.A., SIVER, V.I. Regeneration of used vegetable oil by trysodium phosphate and ammonia gas. Rybnoe Khozyaistvo. Krasnodar., USSR, n. 6, p. 70-71, 1983.

5 COHEN, M.D. Frying oil purification. US. 4, 235, 795, 1980 .

6 COSTA NETO, P.R. Estudos preliminares sobre alterações do óleo de soja usado em frituras múltiplas. Curitiba, 1993. 114 p. Dissertação (Mestrado em Alimentos) Curso de Pós-Graduação em Tecnologia Química, Universidade Federal do Paraná.

7 FRIEDMAN, B. Fryer oil treatment process. UK, 2, 127, 035 A. 1984.

8 GIOIELLI, L.A., PITOMBO, R.N.M., SAKATE, M.S. Efeito da atividade de água na formação de compostos voláteis durante a oxidação de óleo de amendoim bruto. Ciência e Tecnologia de Alimentos, Campinas, v. 12, n. 1, p. 11-98, jan./jun., 1992 .

9 GUTCHO, M. Edible oil and fats. Park Ridge : Noyes Data Corporation, 1979.

10 HAMADA, A., OOHIRA, K. Oil regeneration membrane. UK. 2, $080,350 A, 1982$. 
11 JACOBSON, A.G. Quality control in deep-fat frying operations. Food Technology, p.72-74, Feb., 1991.

12 KOVAL, E.I., MOISEEVA, V.G., ZAIKO, G.M., ANAN EVA, M.K. Regeneration of spent vegetable oil resulting from frying vegetables. USSR. 1, 120, 016, A. 1984.

13 NORMAM, N., POTTER, N. La ciencia de los alimentos. México : Centro Regional de Ayuda Técnica, 1973.

14 PARANÁ. Secretaria de Estado da Agricultura. Empresa paranaense de classificação de produtos (CLASPAR) . Resolução do Concex n.169 de 08/03/1989. Curitiba, 1989.

15 PENJES, J. Experimental studies on decoloration and subsequent filtration of soybean oil. FSTA., Belgium, v. $9,6 \mathrm{n} 313,1977$.

16 REGUTTI, R. Method of marking a composite filter material and its use in treating edible oils. US, 5, 077, 070, 1991.

17 SÃO PAULO. Instituto Adolfo Lutz. Normas analíticas do Instituto Adolfo Lutz (IAL). São Paulo, 1976. 371 p.

18 SÃo PAULO. Secretaria da Indústria Comércio Ciência e Tecnologia. Soja: produção, pré-processamento e transformação agroindustrial. São Paulo : Secretaria da Indústria Comércio Ciência e Tecnologia, [199-]. 99 p. Série Extensão Agroindustrial, 7.

19 TAYLOR, D.R., GALLAVAN, K.P. Treatment of impure frying oil. US, 4, 735, 815, 1988.

20 TARAN, N.G., BEZGUDOVA, Zh. I., SHEVCHENKO, I.B., ARTEMENKO, T.P. Clarification of vegetable oils using kerch bentonite clays. Izvestiya vysshikhuchebnykh zavedenii, pishchevaya tekchnologiva., Odessa (USSR), n. 4, p. 154-156, 1973 .

21 WHALEY, B.M., WHALEY, J.C. Methods for filtering frying oil. US, 4, 328, 097, 1982 .

22 WHALEY, B.M., WHALEY, J.C. Apparatus for frying oils. US, 4, 328, 097, 1982 . 